\title{
Carcinoma de Células Escamosas da Boca: Concordância Diagnóstica em Exames Realizados no Laboratório de Anatomia Patológica da Universidade Federal de Alfenas
}

Squamous Cell Carcinoma of the Mouth: Diagnostic Agreement in Tests Performed in the Laboratory of Pathological Anatomy at the Federal University of Alfenas Carcinoma de Células Escamosas de la Boca: Conformidad Diagnóstica en Pruebas Realizadas en Laboratorio Clínico de Anatomía Patológica de la Universidad Federal de Alfenas

\author{
Antônio Camilo de Souza Cruz'; Solange de Oliveira Braga Franzolin²; Alessandro Antônio Costa Pereira³; Luiz Alberto Beijo4; \\ João Adolfo Costa Hanneman ${ }^{5}$; Júlia Rosental de Souza Cruz ${ }^{6}$
}

\section{Resumo}

Introduçáo: $\mathrm{O}$ câncer da cavidade bucal está entre as dez neoplasias mais frequentes na população brasileira. É diagnosticado na maioria dos casos em estágios avançados, dificultando o tratamento e reduzindo o índice de sobrevida dos pacientes. Objetivo: Aferir a concordância entre os diagnósticos clínicos, histopatológicos das biópsias e das peças cirúrgicas do carcinoma de células escamosas da boca. Método: Realizou-se um estudo retrospectivo entre janeiro de 2000 e dezembro de 2010, utilizando laudos histopatológicos emitidos pelo Laboratório de Anatomia Patológica da Universidade Federal de Alfenas, Minas Gerais, Brasil e os prontuários hospitalares dos pacientes tratados. Para que fosse determinada a concordância, os diagnósticos clínicos foram confrontados com os histopatológicos da biópsia e estes com o exame das peças cirúrgicas removidas no tratamento. Resultados: A maior incidência de CCE ocorreu em pacientes brancos, gênero masculino, com idade entre 50 a 60 anos, solteiros, lavradores ou domésticas, cursaram apenas o ensino fundamental, baixo nível socioeconômico, tabagistas e/ou etilistas. Os sítios anatômicos mais comuns das lesôes foram assoalho bucal e língua. O vestíbulo da boca e o palato foram os sítios menos frequentes. A concordância entre os diagnósticos clínico/histopatológico foi de $71,2 \%$ e entre o histopatológico e o exame da peça cirúrgica 87,1\%. Conclusão: Observou-se expressiva concordância entre o diagnóstico. Entretanto, são necessários novos levantamentos que comparem o resultado dos exames da peça cirúrgica removida no tratamento com as características clínicas. Palavras-chave: Biópsia; Patologia Bucal; Diagnóstico Clínico; Carcinoma de Células Escamosas; Minas Gerais

\footnotetext{
${ }^{1}$ Professor Adjunto da Disciplina de Patologia. Departamento de Patologia e Parasitologia. Instituto de Ciências Biomédicas da Universidade Federal de Alfenas (Unifal). Alfenas (MG), Brasil. Doutorando em Biologia Oral pela Universidade Sagrado Coração (USC). Bauru (SP), Brasil.

${ }^{2}$ Professora-Assistente da Disciplina de Bioestatística da USC. Bauru (SP), Brasil. Doutora em Fisiopatologia em Clínica Médica pela Universidade Estadual Paulista (UNESP).

${ }^{3}$ Professor-Associado da Disciplina de Patologia. Departamento de Patologia e Parasitologia. Instituto de Ciências Biomédicas da Unifal. Alfenas (MG), Brasil. Doutor em Odontologia pela Universidade de São Paulo (USP).

${ }^{4}$ Professor Adjunto da Disciplina de Bioestatística do Instituto de Ciências Exatas da Unifal. Alfenas (MG), Brasil. Doutor em Estatística e Experimentação Agropecuária pela Universidade Federal de Lavras (UFLA). Lavras (MG), Brasil.

${ }^{5}$ Professor Adjunto da Disciplina de Estomatologia da Faculdade de Odontologia da Unifal. Alfenas (MG), Brasil. Doutor em Odontologia pela USP.

${ }^{6}$ Acadêmica de Nutrição da Unifal. Alfenas (MG), Brasil.

Endereço para correspondência: Antônio Camilo de Souza Cruz. Departamento de Patologia e Parasitologia. Instituto de Ciências Biomédicas da Universidade Federal de Alfenas. Rua Gabriel Monteiro da Silva, 700. Alfenas (MG), Brasil. CEP:37130-000. E-mail: camilocruz@terra.com.br.
} 


\section{INTRODUÇÃO}

O câncer da boca está entre os dez tipos de neoplasias mais frequentes na população brasileira, sendo o quinto mais incidente em homens e o sétimo em mulheres. Para 2012, estatísticas válidas também para 2013, há um risco estimado de dez casos novos a cada 100 mil homens e quatro a cada 100 mil mulheres ${ }^{1}$. As taxas de incidência e mortalidade são preocupantes, estando entre as mais elevadas do mundo ${ }^{2}$, com o carcinoma de células escamosas (CCE) correspondendo de $90 \%$ a $95 \%$ dos casos 3 .

O CCE da boca apresenta variadas formas clínicas, incluindo exofíticas, endofíticas, leucoplásicas, eritroplásicas ou eritroleucoplásicas, assim como três padróes de crescimento: exofítico, ulcerativo e verrucoso ${ }^{4}$. Histologicamente, apresenta-se como uma proliferação de células da camada espinhosa, que se dispóe em grupos celulares formando cordóes e ninhos, ou de forma individual invadindo o tecido conjuntivo ${ }^{4}$.

O diagnóstico clínico de uma lesão é estabelecido em função de uma série de dados obtidos por meio de anamnese e exame físico; havendo suspeita de neoplasia, a biópsia é obrigatória para definição do caso pelo exame histopatológico 5 . O cirurgiáo-dentista deve estar apto a realizar a indicação adequada da biópsia e tratar adequadamente o material colhido ${ }^{6}$. A biópsia com bisturi é invasiva e tem potencial de morbidade, devendo ser reservada para avaliação de lesôes altamente suspeitas ${ }^{7}$. Silveira et al. ${ }^{8}$ recomendam atenção especial por parte do clínico, especialmente as eritroplasias; que, em seus estudos, apresentaram um elevado percentual de malignização e o maior índice de equívocos no diagnóstico clínico. Em Porto Rico, as biópsias são realizadas com maior frequência em casos de carcinoma invasivo, indicando deficiência na indicação para lesões pré-cancerosas ${ }^{9}$. Dentistas do Estado de Nova Iorque estâo bem informados sobre câncer oral, mas existem lacunas no conhecimento de certos fatores de risco e na técnica de exame bucal ${ }^{10}$.

A concordância diagnóstica foi avaliada por vários estudos: Marin et al. ${ }^{6}$ registraram mais da metade dos diagnósticos clínicos e histopatológicos concordantes. Vier et al. ${ }^{11}$ relacionaram diagnóstico clínico ao histopatológico e registraram $79,9 \%$ de concordantes. Alves et al. ${ }^{12}$ revisaram 1.825 laudos e, em $67,05 \%$, o diagnóstico clínico concordava com o histopatológico. Aquino et al. ${ }^{13}$ aferiram a concordância entre as três primeiras hipóteses clínicas e histopatológicas. Em 78,5\% dos diagnósticos clínicos, houve concordância com o histopatológico, sendo 57,0\% na primeira hipótese, $19 \%$ na segunda e 2,5\%, na terceira.

$\mathrm{Na}$ área médica, também são encontrados alguns estudos que comparam diagnósticos clínicos e histopatológicos, em que a concordância varia de acordo com o grau de dificuldade de obtenção do diagnóstico clínico ou com os critérios de estabelecimento de determinados diagnósticos ${ }^{14}$.

Procurando contribuir com novos dados sobre o tema, o presente estudo tem por objetivo aferir a concordância entre os diagnósticos clínicos, histopatológicos das biópsias e das peças cirúrgicas do carcinoma de células escamosas da boca.

\section{MÉTODO}

O projeto de pesquisa foi aprovado pelo Comitê de Ética em Pesquisa da Universidade Sagrado Coração em 28/10/2010, sob o n ${ }^{\circ} 197 / 2010$.

O estudo consiste de um levantamento de dados dos pacientes com diagnóstico de carcinoma de células escamosas da boca realizado no Laboratório de Anatomia Patológica da Universidade Federal de Alfenas (Unifal-MG), de janeiro de 2000 a dezembro de 2010. Foram incluídos todos os pacientes que tiveram seu prontuário odontológico e/ou hospitalar localizado. A pesquisa dos prontuários foi realizada nos hospitais: Bom Pastor em Varginha (MG), Santa Casa de Alfenas (MG) e Santa Casa de Poços de Caldas (MG), onde os pacientes foram atendidos.

Dos laudos anatomopatológicos, prontuários odontológicos e hospitalares foram coletados: idade, gênero, cor da pele, estado civil, escolaridade, profissáo, nível socioeconômico, consumo de álcool e/ou tabaco, localização da lesão, tempo de evoluçáo, diagnóstico clínico sugerido pelo profissional, diagnóstico histopatológico da biópsia, tratamento instituído e resultado do exame da peça cirúrgica removida no tratamento. Os diagnósticos clínicos foram confrontados com os diagnósticos histopatológicos da Unifal-MG e estes com o exame das peças cirúrgicas para que fosse determinada a concordância. Quando clinicamente foi apresentada mais de uma possibilidade diagnóstica, a concordância foi aferida pela primeira opção sugerida pelo cirurgião-dentista.

Para a definição da localização anatômica, foi adotada como referência a Classificação Internacional de Doenças - 10a edição (CID-10) da Organização Mundial da Saúde (OMS) de CCE. Foram considerados neste estudo os seguintes sítios anatômicos: lábio (CID.C00), língua oral (CID.C02), rebordo gengival (CID.C03), assoalho da boca (CID.C04), palato (CID.C05), mucosa jugal (CID. C06.0), vestíbulo da boca (CID C06.1) e área retromolar (CID.C06.2).

Os testes estatísticos foram realizados com o programa BioEstat 5.0. Utilizou-se estatística descritiva (frequência absoluta - n; frequência relativa - \%) e inferencial (Teste Exato de Fisher e o Teste Qui-Quadrado) para analisar as variáveis, adotando-se o índice de confiabilidade de 95\%. 


\section{RESULTADOS}

As biópsias foram provenientes em sua maioria (77\%) das clínicas de Estomatologia da Universidade Federal de Alfenas, Universidade José do Rosário Velano e Universidade do Vale do Rio Verde. Estomatologistas e cirurgióes maxilofaciais foram responsáveis pelo envio de $18 \%$ dos casos e os clínicos, por apenas $5 \%$. Os CCE diagnosticados correspondem a 2,33\% das lesóes examinadas no período considerado no estudo, pelo Laboratório de Anatomia Patológica da Unifal-MG (Tabela 1). O estudo incluiu 60 pacientes, a maioria brancos, gênero masculino, com idade entre 50 a 60 anos, solteiros, lavradores ou domésticas, cursaram apenas o ensino fundamental, baixo nível socioeconômico, tabagistas e/ou etilistas. Apenas um caso chegou ao laboratório sem o diagnóstico clínico, não sendo considerado para estabelecer a concordância diagnóstica.

A Tabela 2 demostra que a maioria dos CCE localizava-se no assoalho bucal e na língua. $\mathrm{O}$ vestíbulo da boca e o palato foram os sítios de menor frequencia. Não houve divergências entre os diagnósticos clínicos e histopatológicos para as lesôes localizadas na mucosa jugal. A maior frequência foi observada para as lesóes localizadas no palato $(66,7 \%)$ e na língua $(46,2 \%)$.

$\mathrm{Na}$ Tabela 3, verifica-se que, entre os diagnósticos clínicos e histopatológicos, houve concordância em 71,2\% dos casos. As patologias citadas equivocadamente com maior frequência pelos cirurgiōes dentistas no diagnóstico clínico foram: leucoplasia, carcinoma verrucoso, hiperplasia, paracoccidioidomicose e úlcera traumática.

Tabela 1. Exames realizados pelo serviço de anatomia patológica da Unifal-MG

\begin{tabular}{c|c|c|c|c}
\hline \multirow{2}{*}{ Ano } & \multirow{2}{*}{ Exames realizados } & \multicolumn{2}{|c|}{ CCE diagnosticados } & Pacientes localizados \\
\cline { 3 - 5 } & & Número & $\%$ & 3 \\
\hline 2000 & 347 & 06 & 1,73 & 1 \\
\hline 2001 & 225 & 02 & 0,89 & 0 \\
\hline 2002 & 258 & 02 & 0,78 & 4 \\
\hline 2003 & 284 & 06 & 2,11 & 2 \\
\hline 2004 & 282 & 03 & 1,06 & 5 \\
\hline 2005 & 308 & 05 & 1,62 & 2 \\
\hline 2006 & 388 & 06 & 1.55 & 3 \\
\hline 2007 & 347 & 08 & 2,31 & 7 \\
\hline 2008 & 367 & 10 & 2,72 & 12 \\
\hline 2009 & 381 & 13 & 3,41 & 21 \\
\hline 2010 & 423 & 23 & 5,44 & 60 \\
\hline Total & 3610 & 84 & 2,33 & \\
\hline
\end{tabular}

Tabela 2. Distribuição dos CCE quanto ao sítio anatômico e divergências entre os diagnósticos clínicos, histopatológicos e cirúrgicos

\begin{tabular}{l|c|c|c|c|c|c}
\hline \multirow{2}{*}{ CID } & Localização & No de & \multicolumn{4}{c}{ Número de discordâncias (\%) } \\
\cline { 5 - 7 } & lesões & Clínico/ Histopatológico & $\begin{array}{c}\text { Histopatológico/ Peça } \\
\text { cirúrgica }\end{array}$ \\
\hline CID C00 & Lábio & 5 & 1 & $20,0 \%$ & 1 & $20,0 \%$ \\
\hline CID C02 & Língua oral & 13 & 6 & $46,2 \%$ & 1 & $7,7 \%$ \\
\hline CID C03 & Rebordo gengival & 10 & 3 & $30,0 \%$ & 1 & $10,0 \%$ \\
\hline CID C04 & Assoalho da boca & 17 & 3 & $11,8 \%$ & - & - \\
\hline CID C05 & Palato & 3 & 2 & $66,7 \%$ & 1 & $33,4 \%$ \\
\hline CID C06.0 & Mucosa jugal & 5 & - & - & - & - \\
\hline CID C06.1 & Vestíbulo da boca & 3 & 1 & $33,4 \%$ & - & - \\
\hline CID C06.2 & Área retromolar & 4 & 1 & $25,0 \%$ & - & - \\
\hline Total & & 60 & 17 & & 4 & - \\
\hline
\end{tabular}


Tabela 3. Concordância entre os diagnósticos

\begin{tabular}{|c|c|c|c|c|}
\hline \multirow{2}{*}{$\begin{array}{l}\text { Concordância } \\
\text { diagnóstica }\end{array}$} & \multicolumn{2}{|c|}{ Clínico/Histopatológico } & \multicolumn{2}{|c|}{ Histopatológico/Peça cirúrgica } \\
\hline & $\mathbf{N}^{\circ}$ de pacientes & $\%$ & $\mathbf{N}^{\circ}$ de pacientes & $\%$ \\
\hline Concorda & 42 & 71,2 & 27 & 87,1 \\
\hline Não concorda & 17 & 28,8 & 4 & 12,9 \\
\hline Total & 59 & 100 & 31 & 100 \\
\hline
\end{tabular}

Teste Exato de Fisher $(p=0,1179)$

O tratamento cirúrgico foi aplicado a 31 pacientes, e as lesôes removidas, encaminhadas a diversos laboratórios de patologia, sob a responsabilidade dos hospitais que trataram os pacientes. Na comparação entre os histopatológicos da biópsia diagnóstica e das peças cirúrgicas removidas no tratamento, houve concordância em $87,1 \%$ dos casos, com intervalo de confiabilidade de $83,4 \%$ a $93,8 \%$. Nas divergências, as patologias mais frequentemente apontadas foram: carcinoma verrucoso e hiperplasia.

O $p$ valor $>0,005$ (Tabela 3) evidencia que a concordância entre os diagnósticos clínicos e histopatológicos é estatisticamente igual à concordância destes com o exame das peças cirúrgicas.

\section{DISCUSSÃO}

O percentual de diagnóstico histopatológico de carcinoma de células escamosas da boca $(2,33 \%) \mathrm{em}$ relação ao total de lesóes examinadas na Unifal-MG é muito próximo aos de Kniest et al..$^{15}$ que encontraram frequência de 2,4\%. Resultados semelhantes foram encontrados por Conceição et al. ${ }^{16}$, Daher et al. ${ }^{3}$ e por Silveira et al. ${ }^{8}$ que estudaram lesôes orais com potencial de malignização.

O percentual de concordância diagnóstica (clínico/ histopatológico) desta pesquisa $(71,2 \%)$ se aproxima daqueles encontrados por Conceição et al. ${ }^{16}(76,2 \%)$ para CCE. Lins et al. ${ }^{17}$ determinaram uma maior concordância diagnóstica $(79,3 \%)$ para as neoplasias malignas, quando comparadas a lesões bucais de outra natureza. Os trabalhos de Rados et al. ${ }^{18}$ (46,0\%), Conceição et al. ${ }^{16}$ (57,72\%) e Silva et al. ${ }^{5}(56,67 \%)$ corroboram essa afirmação. No entanto, Aquino et al. ${ }^{13}$ (78,0\%), Alves et al. ${ }^{12}(67,95 \%)$ e Vier et al. ${ }^{11}(79,9 \%)$ encontraram resultados semelhantes para diferentes classes de lesóes bucais. Os números de concordância diagnóstica das lesóes bucais são compatíveis aos determinados para área médica que varia de $44 \%$ a $84 \%$ dependendo do caso ${ }^{14}$. Rados et al. ${ }^{18}$ afirmam que os pós-graduandos têm maior percentual de concordância. Neste estudo, a expressiva maioria dos diagnósticos clínicos foi realizada por especialistas e esse fator pode ter contribuído para aumentar a concordância.
O diagnóstico de CCE da boca pode ser confundido com lesôes ulceradas e sangrantes e/ou de crescimento rápido, principalmente em pacientes com perfil epidemiológico de CCE bucal. Quando ocorre discordância entre os diagnósticos, nem sempre o clínico é incorreto, pois pode ter havido falta de representatividade do material colhido, fixação inadequada ou manipulação imprópria do espécime ${ }^{19}$. Além disso, há uma subjetividade na avaliação diagnóstica do patologista diretamente relacionada a características individuais, independente de sua formação educacional, responsável por expressiva discordância $(31 \%$ a 51\%) na interpretaçáo das lâminas e nos resultados ${ }^{20 ; 21}$. Nestes casos, recomenda-se a comunicação entre o clínico e o patologista para que se possa chegar a um diagnóstico final ${ }^{19}$.

As leucoplasias são lesóes cancerizáveis e podem confundir o clínico se apresentarem áreas avermelhadas. O carcinoma verrucoso é considerado por alguns autores como uma variante do CCE, porém menos agressivo pelo crescimento exofítico, capaz de induzir dúvidas e talvez sendo responsável pela discordância no diagnóstico. As hiperplasias fibrosas inflamatórias, mesmo representando uma proliferação celular, não são consideradas de risco para transformação maligna. No entanto, as ulcerações, especialmente associadas a traumatismo crônico, podem levar o clínico a suspeitar de CCE. Um diagnóstico diferencial é compatível com a paracccidioidomicose pela presença de áreas ulceradas e hemorrágicas, que mesmo quando mais difusas, justificam um diagnóstico diferencial com o CCE. Em todos os casos analisados, a conduta do cirurgião-dentista foi correta, pois a biópsia deve ser realizada sempre que a lesão apresentar características de malignidade 5 .

As diversas lesôes bucais, de acordo com Aquino et al. ${ }^{13}$, localizam-se predominantemente na mucosa jugal e gengiva e, menos frequentemente, no fundo de vestíbulo, enquanto Silva et al. ${ }^{5}$ encontraram maior frequência das lesôes na mucosa jugal e lábio inferior, e os sítios menos acometidos foram o rebordo alveolar superior e mandibular anterior. Este estudo determinou maior incidencia de CCE na língua e no assoalho da boca, coincidindo com os resultados de Oliveira et al. ${ }^{2}$ e menor 
frequencia no palato e vestíbulo da boca, divergindo desses autores que apontaram gengiva e lábio superior.

As divergencias diagnósticas (clínico/histopatológico) ocorreram em percentual mais elevado para as lesóes localizadas no palato $(66,7 \%)$ e na língua $(46,2 \%)$. Conceição et al. ${ }^{16}$ encontraram um elevado percentual $(57,72 \%)$ de divergências para lesôes de língua. Considerando que apenas $15 \%$ dos casos são diagnosticados nos estágios iniciais da lesão e aproximadamente 50\% dos pacientes já chegam aos centros de tratamento com metástases no momento do diagnóstico ${ }^{22}$, há necessidade de maior atenção nos exames clínicos desses sítios anatômicos e melhor formaçáo dos profissionais com relação às características clínicas das lesôes. Nos EUA, cursos de Educação Continuada sobre câncer bucal apresentaram influência positiva sobre as atitudes, conhecimentos e comportamentos dos participantes, o que potencialmente pode fazer a diferença na detecção, prevenção e controle ${ }^{23}$.

As divergências entre os resultados histopatológicos das biópsias e das peças cirúrgicas removidas no tratamento correspondem a 12,9\% dos casos (Tabela 3). As divergências encontradas são relacionadas a carcinoma verrucoso e hiperplasia. Os exames das peças foram realizados em diferentes laboratórios e, portanto, os resultados devem considerar a subjetividade inerente ao patologista. Em algumas patologias, como na paraoccidiodomicose, o epitélio pode mostrar-se com displasias, sendo facilmente confundido com o epitélio do CCE. Nesses casos, é denominado de epitélio com hiperplasia pseudoepiteliomatosa, também descrita em associação a processos inflamatórios crônicos inespecíficos. Para estabelecer o diagnóstico, sugere-se exame de imunofenotipagem para avaliar integridade da membrana basal, possibilitando diagnóstico diferencial de carcinoma verrucoso. O carcinoma verrucoso é classificado como CCE quando ocorrem áreas de microinvasão da lâmina própria. Na peça, o examinador tem oportunidade de preparar cortes de diversas áreas da lesão, podendo constatar que a microinvasividade náo é representativa para classificar o carcinoma verrucoso como CCE. Neste estudo, um diagnóstico clínico de carcinoma verrucoso recebeu classificação histopatológica de CCE na biópsia, sendo posteriormente confirmado como verrucoso no exame da peça cirúrgica removida no tratamento. Portanto, os diagnósticos clínicos e cirúrgicos são, especialmente, relevantes no estabelecimento do diagnóstico final ${ }^{6}$.

É importante destacar que, na literatura, os dados estatísticos relacionando a concordância dos diagnósticos clínico/histopatológico/cirúrgico das lesões bucais são bastante escassos. Considerando as limitaçóes diagnósticas apresentadas por uma biópsia incisional, seria desejável que o cirurgiáo-dentista tivesse acesso aos resultados do exame da peça cirúrgica removida no tratamento, que é mais preciso e detalhado. Essa associação de informações é relevante, uma vez que a sobrevida do paciente é determinada pelo comportamento clínico-patológico do carcinoma ${ }^{24}$.

\section{CONCLUSÃO}

Observou-se expressiva concordância $(71,2 \%)$ entre os diagnósticos clínico e histopatológico da biópsia incisional e destes com o exame da peça cirúrgica $(87,1 \%)$. Para melhor conhecimento das características, comportamento e desenvolvimento do CCE de boca, é desejável que o histórico do paciente portador reúna as informaçóes dos prontuários odontológicos e hospitalares. São necessários novos levantamentos que investiguem os resultados dos exames da peça cirúrgica comparando-os com as informaçôes clínicas.

\section{CONTRIBUIÇÕES}

Antônio Camilo de Souza Cruz contribuiu na concepção e planejamento do projeto de pesquisa, na obtenção e/ou análise e interpretação de dados e na redação e revisão crítica. Solange de Oliveira Braga Franzolin contribuiu na concepçáo e planejamento do projeto de pesquisa e na redação e revisão crítica. Alessandro Antônio Costa Pereira contribuiu na concepção e planejamento do projeto de pesquisa e na obtenção e/ou análise e interpretação de dados. João Adolfo Costa Hanneman e Júlia Rosental de Souza Cruz contribuíram na obtenção e/ou análise e interpretação de dados. Luiz Alberto Beijo contribuiu na obtenção e/ou análise e interpretação de dados e na redação e revisão crítica.

\section{AGRADECIMENTOS}

Aos Registros Hospitalares de Câncer do Hospital Bom Pastor em Varginha (MG), Brasil. Ao Serviço de Oncologia da Santa Casa de Alfenas (MG), Brasil. À Unidade de Assistência de Alta Complexidade em Oncologia de Poços de Caldas (MG), Brasil. À Gerência Regional de Saúde de Alfenas (MG), Brasil. Aos colegas cirurgióes-dentistas e aos profissionais da Área de Saúde que colaboraram direta ou indiretamente para a realização deste trabalho.

\section{Declaraçáo de Conflito de Interesses: Nada a Declarar.}

\section{REFERÊNCIAS}

1. Instituto Nacional de Câncer José Alencar Gomes da Silva. Estimativa 2012: incidência de câncer no Brasil. Rio de Janeiro: INCA; 2011. 118 p. 
2. Oliveira LR, Ribeiro-Silva A, Zucoloto S. Perfil da incidência e da sobrevida de pacientes com carcinoma epidermóide oral em uma população brasileira. Jornal brasileiro de patologia e medicina laboratorial 2006; 42(5): 385-92.

3. Daher GCA, Pereira GA, Oliveira ACD'A. Características epidemiológicas de casos de câncer de boca registrados em hospital de Uberaba no período 1999-2003: um alerta para necessidade de diagnóstico precoce. Rev Bras Epidemiol 2008;11(4): 584-96.

4. Neville BW, Damm DD, Allen CM, Bouquot JE. Patologia Oral e Maxillofacial. 3. ed. Rio de Janeiro: Guanabara-Koogan; 2009. 972 p.

5. Silva TFA, Souza RB, Rocha DR, Araújo FAC, Morais HHA. Levantamento das Biópsias realizadas no serviço de Cirurgia Buco-Maxilo-Facial do Curso de Odontologia da Universidade do Estado do Rio Grande do Norte. Revista de cirurgia e traumatologia buco-maxilo-facial $2011 \mathrm{abr} /$ jun;11(2): 91-100.

6. Marin HJI, Silveira MMF, Souza GFM, Pereira JRD. Lesôes bucais: concordância diagnóstica na Faculdade de Odontologia de Pernambuco. Odontologia clínicocientífica 2007 out/dez; 6(4):315-8.

7. Mehrotra R, Gupta DK. Exciting new advances in oral cancer diagnosis: avenues to early detection. Head Neck Oncol 2011 July; 3:33.

8. Silveira EJD, Lopes MFF, LMM; Ribeiro BF, Lima KC, Queiroz LMG. Lesões orais com potencial de malignização: análise clínica e morfológica de 205 casos. Jornal brasileiro de patologia e medicina laboratorial 2009 jun; 45(3): 233-8.

9. Morse DE, Psoter WJ, Cuadrado L, Jean YA, Phelan J, Mittal K, et al. A deficit in biopsying potentially premalignant oral lesions in Puerto Rico. Cancer Detect Prev 2009; 32(5-6): 424-30.

10. Gajendra S, Cruz GD, Kumar J. Prevention and Early Detection: Knowledge, Practices, and Opinions of Oral Health Care Providers in New York State. J Cancer Educ 2006; 21(3):157-62.

11. Vier FV, Rockenbach MIB, Yurgel LS, Cherubini K, Figueiredo MAZ. Diagnósticos histopatológiาcos do Laboratório de Patologia do Serviço de Estomatologia da PUCRS, nos anos de 2000 a 2002 e sua relação com o diagnóstico clínico. Odonto ciência 2004; 19(46):382-8.

12. Alves JR, Hida M, Nai GA. Diagnóstico clínico e anatomopatológico: discordâncias. AMB Rev Assoc Med Bras 2004; 50(2):178-81.
13. Aquino SN, Martelli DRB, BorgesSP, Bonan PRF, Martelli Junior H. Concordância entre diagnóstico clínico e histopatológico de lesóes bucais. Rev Gaucha Odontol 2010 jul/set; 58( 3): 345-9.

14. Guimarães TC. Correlação clínico-histopatoló gica nos pacientes com hanseníase do anexo de Dermatologia, Professor Oswaldo Costa do Hospital das Clínicas da UFMG entre setembro de 1989 e dezembro de 1998 [dissertação]. Belo Horizonte: Universidade Federal de Minas Gerais; 2001.

15. Kniest G, Stramandinoli RT, Ávila LFC, Izidoro ACS. Frequência das lesōes bucais diagnosticadas no Centro de Especialidades Odontológicas de Tubarão (SC). Revista Sul-brasileira de Odontologia 2011 jan/mar;8(1):13-8.

16. Conceição LD, Magrin T, Gomes APN, Araujo LMA. Estudo retrospectivo de biópsias em língua - aspectos epidemiológicos. RFO 2010 jan/abr; 15(1): 11-9.

17. Lins RC, Simôes CA, Henriques ACG, Cazal C, Castro JFL, Carvalho EJA. Correlação dos índices de acertos entre as hipóteses clínicas e os resultados histopatológicos - clínica versus histopatologia. Int J Dent 2008 jul/set; 7(3):153-7.

18. Sant'Ana Filho M, Barbachan JJD, Volkweis MR, Romanini J. Estudo comparativo da concordância entre o diagnóstico clínico e histopatológico das lesões bucais. Revista da Faculdade de Odontologia de Porto Alegre 1996 jul; 37(1):21-3.

19. Viscomi F. Diagnóstico clínico e diagnóstico anatomopatológico: discordâncias. AMB Rev Assoc Med Bras 2004; 50(2):109-26.

20. Karabulut A, Reibel J, Therkildsen MH, Praetorius F, Nielsen HW, Dabelsteen E. Observer variability in the histologic assessment of oral premalignant lesions. J Oral Pathol Med 1995; 24(5):198-200.

21. Pindborg JJ, Reibel J, Holmstrup P. Subjectivity in evaluating oral epithelial dysplasia, carcinoma in situ and initial carcinoma. J Oral Pathol Med 1985 Out; 14(9): 698-708.

22. Martins RB, Giovani EM, Villalb H. Lesões can ^cerizáveis na cavidade bucal. Revista do Instituto de Ciências da Saúde 2008; 26(4):467-76.

23. Silverman Jr S, Kerr AR, Epstein JB. Oral and Pharyngeal Cancer Control and Early Detection. J Cancer Educ 2010; 25 (3):279-81.

24. Bryne M. Is the invasive front of an oral carcinoma the most important area for prognostication? Oral Dis 1998 Jun; 4(2): 70-7. 


\begin{abstract}
Introduction: Oral cancer is among the ten most frequent neoplasms in the Brazilian population. It is diagnosed in most of the cases in advanced stage, making the treatment harder and reducing the rate of the patient's survival. Objective: Assess the concordance between clinical diagnosis, histopathological biopsies and surgical pieces of the squamous cells carcinoma of the mouth. Method: A retrospective study was carried out from January 2000 to December 2010, based on histophatological reports issued by the Pathological Anatomy Lab from the Federal University of Alfenas, Minas Gerais, Brazil and the hospital records of treated patients. In order to determine the concordance, the clinical diagnoses were compared with the histopathological diagnosis of biopsy and those were compared with the exam from the surgical pieces removed in the treatment. Results: The higher incidence of SCC occurred in white patients, male, aged between 50 and 60, single, farmers or housekeepers, who had attended primary school only, from a low socio-economic level, smokers and/or alcoholics. The most common anatomic sites of the lesions were the floor of the mouth and tongue. The vestibule of the mouth and the palate were the less common sites. The concordance between the clinical and histopathological diagnosis was $71.2 \%$ and between the histopathological and the surgical pieces exam was $87.1 \%$. Conclusion: An expressive concordance between the diagnoses was verified. However, new surveys are necessary that compare the exam results of the surgical pieces during the treatment with their clinical characteristics. Key words: Biopsy; Pathology, Oral; Clinical, Diagnosis; Carcinoma, Squamous Cell; Minas Gerais State
\end{abstract}

\title{
Resumen
}

Introducción: El cáncer de la cavidad bucal está entre las diez neoplasias más comunes en la población brasileña. En la mayoría de los casos es diagnosticado en etapas avanzadas, dificultando el tratamiento y disminuyendo la tasa de supervivencia de los pacientes. Objetivo: Evaluar la correlación entre los diagnósticos clínicos, histopatológicos de las biopsias y de las piezas quirúrgicas del carcinoma de células escamosas de la boca. Método: Se realizó un estudio retrospectivo de enero de 2000 y diciembre de 2010, utilizando informes histopatológicos emitidos por el Laboratorio de Anatomía Patológica de la Universidad Federal de Alfenas, Minas Gerais, Brasil y la historia clínica de los pacientes tratados. Para que sea determinada la correlación, los diagnósticos clínicos han sido comparados con los histopatológicos de la biopsia y esos con la prueba de las piezas quirúrgicas removidas en el tratamiento. Resultados: La mayor incidencia del CCE ocurrió en pacientes blancos, género masculino, con edad entre 50 y 60 años, solteros, agricultores o empleados del hogar que sólo cursaron la educación primaria obligatoria, bajo nivel socioeconómico, fumadores y/o alcohólicos. Los sitios anatómicos más comunes de las lesiones fueron piso de la boca y lengua. El vestíbulo de la boca y el paladar han sido los sitios con menor frecuencia. La concordancia entre los diagnósticos clínico/histopatológico fue de $71,2 \%$ y entre el histopatológico y la prueba en la pieza quirúrgica $87,1 \%$. Conclusión: Se observó una concordancia significativa entre el diagnóstico. Sin embargo, son necesarios nuevos estudios que comparen el resultado de las pruebas en la pieza quirúrgica eliminada en el tratamiento con las características clínicas. Palabras clave: Biopsia; Patología Bucal; Diagnóstico Clínico; Carcinoma de Células Escamosas; Minas Gerais 